\title{
Cryo-electron Tomography Workflows for Quantitative Analysis of Actin Networks Involved in Cell Migration
}

Florian Fäßler ${ }^{1}$, Georgi Dimchev ${ }^{1}$, Victor-Valentin Hodirnau ${ }^{1}$, Bettina Zens ${ }^{1}$, Christoph Möhl ${ }^{2}$, Frank Bradke $^{2}$ and Florian Schur ${ }^{1 \#}$

${ }^{1}$ Institute of Science and Technology Austria, Klosterneuburg, Niederosterreich, Austria, ${ }^{2}$ German Center for Neurodegenerative Diseases (DZNE), Bonn, Nordrhein-Westfalen, Germany

${ }^{\#}$ Corresponding author

Deriving robust quantitative parameters from cellular processes often requires the combination of different experimental techniques to provide a holistic description of underlying biological mechanisms. In particular, dynamic cellular processes, such as cell migration, benefit from the combination of quantitative multi-scale experimental approaches (i.e. from the micrometer to nanometer scale), including both morphological analysis as well as ultrastructural characterization. Cryo-electron tomography (cryo-ET) is well-suited for native ultrastructural characterization because of its ability to visualize unperturbed cellular environments [1]. The challenge, however, lies in the extraction of quantitative parameters from sufficiently large cryo-ET datasets due to difficulties in specimen preparation, data acquisition, low signal to noise ratio inherent to cryo-ET, and the complexity of cellular data.

Among the biological systems that heavily benefit from quantitative measures are the molecular machineries underlying cell migration. A key player in directed cell migration in eukaryotic cells is the actin cytoskeleton and its associated regulatory proteins [2]. Tight regulation of actin polymerization is required for the formation of higher-order actin assemblies, which mediate essential processes. Such higher-order cellular actin structures include lamellipodia or filopodia, sheet- or finger-like extensions densely filled with actin at the leading edge of migrating cells [3]. Ultrastructural insights into these assemblies combined with extensive characterization of morphology and underlying cellular dynamics of wildtype or genetically modified cells can provide an accurate description of the role of selected players in initiation and maintenance of these actin networks. Obtaining enough cryo-ET data required for statistical relevance has, however, been hindered by the above-mentioned experimental challenges ranging from sample preparation to data acquisition and image analysis.

In order to overcome these challenges, we have developed a workflow that aims at improving several of the steps involved. This includes implementation of custom-designed electron microscopy grid holders for cell culture experiments to allow reproducible and easy-to-handle sample preparation of diverse sensitive cellular specimens. With increased automation and speed of data acquisition we can obtain datasets of several dozens to hundreds of tomograms, which provide the basis for a sufficiently large sample size for statistically sound quantitative conclusions. Such dataset sizes cannot, however, be manually annotated due to the considerable time required for analysis. In order to analyze and interpret these large amounts of data, we have implemented the deep learning tool YAPIC (https://yapic.github.io/yapic) to perform neural network-assisted segmentation of actin filaments in cellular cryo-ET data. By following segmentation with automatic actin filament tracking algorithms using the localized radon transform [4], a vector-based representation of filaments allows analyzing filament population subsets for defined parameters such as filament length, angle and curvature, as well as their orientation to the cellular edge. Besides filament parameters, we also aim for semi-quantitative protein 
detection in lamellipodia via template matching approaches to approximate stoichiometric numbers of proteins involved in regulation and maintenance of actin-based protrusions.

We have successfully applied this workflow to migratory wildtype and knockout cells lacking selected actin regulatory proteins to compare their ultrastructural actin network topologies. Hence, using this data, we can provide a new level of detail about how the presence and activity of individual proteins influence arrangement of actin networks, shedding more light on their specific function. In summary, performing in situ structural biology experiments and relating ultrastructural observations in a native environment to a protein's function is one of the key promises of cryo-ET. Our workflow to describe actin architecture in cell migration using cryo-ET is one example of how this method can drive our understanding of yet unresolved cell biological mechanisms.

\section{References}

1. Asano, S., Engel, B. D. \& Baumeister, W. In Situ Cryo-Electron Tomography: A Post-Reductionist Approach to Structural Biology. J. Mol. Biol. 428, 332-343 (2016). doi:10.1016/J.JMB.2015.09.030

2. Blanchoin, L., Boujemaa-Paterski, R., Sykes, C. \& Plastino, J. Actin Dynamics, Architecture, and Mechanics in Cell Motility. Physiol. Rev. 94, 235-263 (2014). doi:10.1152/physrev.00018.2013

3. Rottner, K. \& Schaks, M. Assembling actin filaments for protrusion. Curr. Opin. Cell Biol. 56, 53-63 (2019). doi:10.1016/J.CEB.2018.09.004

4. Winkler, C., Vinzenz, M., Small, J. V. \& Schmeiser, C. Actin filament tracking in electron tomograms of negatively stained lamellipodia using the localized radon transform. J Struct Biol 178, 19-28 (2012). doi:http://dx.doi.org/10.1016/j.jsb.2012.02.011 